\title{
PTU-024 COLONOSCOPIC SURVEILLANCE AND FOLLOW UP IN PATIENTS WITH ACROMEGALY
}

doi:10.1136/gut.2011.239301.152

N Tehami, S Dover Department of Gastroenterology, Greater Glasgow \& Clyde NHS Trust, Glasgow, UK

Introduction The prevalence of colonic polyps and cancer in patients with acromegaly group has remained controversial despite some earlier reports which regarded this group of patients as high risk for developing colonic cancer.

Methods Patients were identified from pituitary data base and endoscopy records between year 1998 and 2009. Data on referral for screening colonoscopy, completion rates, quality of bowel preparation, examination findings and follow up arrangements was obtained and retrospective data analysis was performed.

Results 48 patients were identified. $40 / 48$ patients had a screening colonoscopy. $6 / 8$ patients were not referred and $2 / 8$ patients did not attend the appointments. 40 patients comprised of 21 Men:19 women, Mean age was 60 years at first colonoscopy (range 22-89). All patients were age more than 40 years except one (age 22 years). Mean age at diagnosis was 44 year (range 22-77). Mean delay in referral for screening colonoscopy from the time patient turned 40 and taking into account the age at diagnosis was 12.26 years (range $0-40$ years).

A total of 78 colonoscopies were performed. 20 patients had at least one surveillance colonoscopy. Indication of colonoscopy was screening/surveillance for polyps including 4 cases with altered bowel habits and anaemia. The time taken to complete the examination was not documented. All the procedures were performed by a single colonoscopist. 69/78 (88\%) procedures had satisfactory bowel preparation with standard regime. Overall 72/78 (92\%) of patients had a complete examination with adequate preparation. The reason for incomplete examination was excessive looping in four patients and inadequate bowel preparation in two cases. No complications relating to colonoscopy occurred.

49/78 (63\%) normal colonic examination including nine patients with diverticulosis, two patients with melanosis and one each with angiodysplasia and a lipoma in the caecum. $29 / 78$ (37\%) of patients had polyps, Of these 10/78 (13 \%) had adenomatous polyps, none were severely dysplastic, no cancer detected.

26/40 (65\%) patients are being followed up as per BSG guidelines. Out of 14 patients who are not being followed up 1 patient has died recently, 1 patient is being followed up with 
a CT pneumocolon, 1 patient has DNA follow up colonoscopy. $11 / 20(27.5 \%)$ patients did not have a follow up colonoscopy or CT pneumocolon.

Conclusion There was a significant delay before patients are referred for screening colonoscopy. The prevalence of adenomatous colonic polyps and cancer in acromegaly patients was not very significant. Unfortunately a significant proportion of these patients were not being followed up as recommended by BSG.

Competing interests None.

\section{REFERENCE}

1. Cairns SR, Scholefield JH, Steele RJ, et al. Guidelines for colorectal cancer screening and surveillance in moderate and high risk groups (update from 2002). Gut 2010;59:666-89 